\title{
OFFSHORE STRUCTURE MODELING
}




\section{ADVANCED SERIES ON OCEAN ENGINEERING}

Series Editor-in-Chief

Philip L- F Llu (Cornell University)

Vol. 1 The Applied Dynamics of Ocean Surface Waves by Chiang $C$ Mei (MIT)

Vol. 2 Water Wave Mechanics for Engineers and Scientists by Robert $G$ Dean (Univ. Florida) and Robert A Dalrymple (Univ. Delaware)

Vol. 3 Mechanics of Coastal Sediment Transport by Jergen Fredsere and Rolf Deigaard (Tech. Univ. Denmark)

Vol. 4 Coastal Bottom Boundary Layers and Sediment Transport by Peter Nielsen (Univ. Queensland)

Vol. 5 Numerical Modeling of Ocean Dynamics by Zygmunt Kowalik (Univ. Alaska) and T S Murty (Inst. Ocean Science, BC)

Vol. 6 Kalman Fitter Method in the Analysis of Vibrations Due to Water Waves by Piotr Wilde and Andrzej Kozakiewicz (Inst. Hydroengineering, Polish Academy of Sciences)

Vol. 7 Physical Models and Laboratory Techniques in Coastal Engineering by Steven A. Hughes (Coastal Engineering Research Center, USA)

Vol. 8 Ocean Disposal of Wastewater by lan $R$ Wood (Univ. Canterbury), Robert $G$ Bell (National Institute of Water \& Atmospheric Research, New Zealand) and David L Wilkinson (Univ. New South Wales)

Vol. 9 Offshore Structure Modeling by Subrata K. Chakrabarti (Chicago Bridge \& Iron Technical Services Co., USA)

Forthcoming titles:

Water Waves Propagation Over Uneven Bottoms by Maarten W Dingemans (Delft Hydraulics)

Tsunami Run-up

by Philip L-F Liu (Cornell Univ.), Costas Synolakis (Univ. Southern California), Harry Yeh (Univ. Washington) and Nobu Shuto (Tohoku Univ.)

Beach Nourishment: Theory and Practice by Robert $G$ Dean (Univ. Florida)

Design and Construction of Maritime Structures for Protection Against Waves by Miguel A Losada (Univ. da Cantabria) and Nobuhisa Kobayashi (Univ. Delaware) 
Advanced Series on Ocean Engineering - Volume 9

\section{OFFSHORE STRUCTURE MODELING}

\section{SIBRATA KUMAR CHAKRABARTI}

Chicago Bridge \& Iron Technical Services Co.

Plainfield, Illinois

USA 
Published by

World Scientific Publishing Co. Ptc. Led.

P O Box 128, Farrer Road, Singapore 9128

USA office: Suite 1B, 1060 Main Street, River Edge, NJ 07661

UK office: 73 Lynton Mead, Totteridge, London N20 8DH

\section{OFFSHORE STRUCTURE MODELING}

Copyright $\odot 1994$ by World Scientific Publishing Co. Pte. Ltd.

All rights reserved. This book, or parts thereof, may not be reproduced in any form or by any means, electronic or mechanical, including photocopying, recording or any information storage and retrieval system now known or to be invented, without written permission from the Publisher.

For photocopying of material in this volume, please pay a copying fee through the Copyright Clearance Center, Inc., 27 Congress Street, Salem, MA 01970, USA.

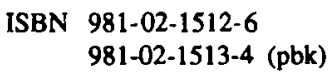

Printed in Singapore by JBW Printers \& Binders Pte. Ltd. 


\section{ACKNOWLEDGEMENTS}

Since joining CBI twenty-five years ago I have been involved in model testing of offshore and marine structures. Many colleagues of mine have helped me understand tricks of model testing over the years. In particular, Erik Brogren provided guidance on model construction details and Alan Libby explained intricacies of instrumentation design.

Many experts reviewed the chapters of this book. Dr. Devinder Sodhi and Prof. Tom Dawson reviewed Chapter 2. Prof. Dawson also checked sections of Chapter 7. Dr. E.R. Funke reviewed Chapter 4. Prof. Christian Aage took the time to review Chapters 4, 7 and 10 and provided valuable comments. Chapter 5 was reviewed by Prof. Bob Hudspeth, Dr. E. Mansard, and Dr. Andrew Cornett. Dr. Erling Huse commented on Chapter 6. Chapter 7 was read by Prof. Li and Dr. Ove Gudmestad. Prof. S. Bhattacharyya improved on Chapter 8. Chapter 9 was reviewed by Dr. O. Nwogu. Keith Melin again reviewed the entire book and provided many editorial and other comments which improved its quality. I am grateful to these individuals. I am, however, responsible for any shortcomings in this book.

CBI Technical Services provided the secretarial help which made this book possible. Many individuals helped in putting the manuscript together, of which the most noteworthy is Ms. Danielle Cantu who finalized the manuscript in its printed form by retyping and reformatting it many times. Finally, I acknowledge the patience of my wife Prakriti (Nature) for providing me the time at home over the last 3 years to complete the book. 
This page is intentionally left blank 


\section{DEDICATION}

This third book by the author is dedicated with pride to the following people that had the greatest contribution in shaping his professional life.

- His professor and Ph. D. thesis adviser, Dr. William L. Wainwright who gave him the first lessons in writing a technical paper which became the first publication by the author.

- His first supervisor, Mr. William A. Tam, then Director of Marine Research, who had faith in the author's ability and gave him an opportunity to work on hydrodynamic related subjects.

- Dr. Basil W. Wilson, then consultant of Chicago Bridge and Iron Co. who taught the author many of the basics of ocean engineering and mooring systems. The author had the good fortune then in cooperating with Dr. Wilson on several research projects. 
This page is intentionally left blank 


\section{PREFACE}

The offshore industry has matured over the years through innovation, initiatives and experience. The industry has advanced a long way to its present stage from its first installation of an exploration structure in coastal waters of the Gulf of Mexico in the 1940 's. The early structures were fixed to the ocean floor and looked much like the electrical transmission towers common on land. Today the shape, size and type of offshore structures vary depending on the required applications. Offshore structures are abundant in all parts of the world. In addition to structures fixed at their base, moored floating structures and vertically tethered structures have been installed for exploration, production, storage and offshore processing of crude oil. While offshore exploration has been relatively trouble free, there have been a few catastrophic failures. Therefore, design of these structures for accident-free operation under anticipated conditions is vital for the continued success and growth of the offshore industry. One of the means of verifying the design of a structure is the testing of scale model of the structure in a simulated ocean environment during its design phase.

Model testing has been performed through the history of mankind. Systematic hydraulic scale model testing goes back to the nineteenth century. However, even today modeling is partly an art as well as a science. In modeling, certain laws of similarity are followed. Several text books are available that deal with these similarity laws. In many cases, these laws can not all be satisfied in a model test. In these cases, it is necessary to selectively distort some of these scaling laws to perform the model tests. Although this distortion is somewhat of a compromise, valid modeling results can still be expected.

While the technical literature dealing with offshore structures discusses model testing, a comprehensive book in this area discussing design, construction, instrumentation, testing and analysis of physical model is lacking. It is desirable that a single text contain the theoretical and practical aspects of physical modeling. Such a book should be valuable to engineers dealing with the design, construction, installation and operation of offshore structures. This requirement inspired me to write this book on modeling. This book provides reasonably detailed coverage of the technology of model testing. As such, it has applications throughout the entire field of engineering, reaching far beyond its focus on offshore structures. It should be equally appealing to engineers and scientists involved with the design and construction of unique structures.

The Introduction discusses the general need for model testing. A brief history of testing has been given in this section. Some of the general structural areas where model testing has been required have also been mentioned. Chapter 2 describes the modeling laws. It begins with the general requirements for similarity. A general discussion of the famous Buckingham pi theorem and an application of the pi theorem has been included. A few specific examples of modeling are discussed here including structural modeling, testing in uniform flows and modeling distortion. These are considered unique cases that 
may be applicable to an offshore structure. In hydraulic testing, however, Froude model law is discussed in detail in this section. A few textbooks that deal with the similarity laws and model testing have been discussed and referenced here. The methods of model construction are considered in Chapter 3. The physical requirements of the model necessary for scale testing are explained. Generally, the construction technique varies based on these requirements. For example, the fixed structures are generally used in the measurement of loads and stresses imposed by the environment. In this case, the dynamic properties of the structure are not a concern. This, however, is not true for compliant or floating structures. The compliant structure, in addition, must satisfy additional scaling laws. The static and dynamic properties that must be satisfied by these structures are discussed in detail. The techniques used in verifying these properties before testing can take place are illustrated.

The testing of offshore structures requires specialized facilities. Many of the small facilities that exist at the universities and other educational institutions are used as teaching tools in discussing the needs and methods of model testing. However, many larger commercial facilities are in existence in various parts of the world. Many of these facilities are described in detail in Chapter 4 including their capabilities and limitations. This section will be useful to a design engineer in choosing a suitable facility for his particular test requirements. The important components of these testing facilities, such as the wave generators, the current generators, the towing carriages and beaches are described. The most important feature of these testing facilities is the wave generation capability. A few theories of wave generation and beach reflection are presented for those interested in designing wavemakers. These facilities are used in duplicating the ocean environment. The modeling of the ocean environment is the subject of Chapter 5 . Simulation of various types of waves, such as random two-dimensional and threedimensional waves, wave groups, and higher harmonic waves, are discussed in detail. The wind and current generation and co-generation of current and waves are also explained in this section. Another important requirement of any model testing is the measurement of the responses of a structure model. This includes the inputs to structures from external sources and the corresponding outputs. In hydraulic testing, this measurement is further complicated by the presence of fluids. Various types of measuring instruments and measuring techniques are introduced in Chapter 6. Design methods of a few specialized instruments are described and methods of the waterproofing these instruments are discussed. The calibration procedure for special instruments is shown. Typical calibration curves of a few of these are also included. The important considerations in the recording of data output from these instruments are given here.

The actual modeling of various offshore structures is described in Chapters 7-9. Various areas covered in these chapters are outlined in the following: 
EXED STRUCTURES

Gravity Platform

Storage Structures

Piled Jackets

Subsea Pipelines

\section{OEESHORE OPERATIONS}

Transport of Jackets

Towing of Structures

Launching of Structures

Submergence of Structures

Pipelaying

\section{ELOATING STRUCTURES}

\author{
Buoys \\ Single Point Moorings \\ Moored Tankers \\ Tension Leg Platforms \\ Compliant Structures \\ Semisubmersibles
}

Chapter 7 deals with the fixed structures. The methods of installing load measuring devices on fixed structures and associated problems are described in detail. Various examples are included. Actual recorded data are given to illustrate the validity as well as inadequacies of the techniques. Offshore operations include special techniques in delivering the completed structure to the offshore sites. These require launching, transportation, submergence and installation of these structures. These various stages are generally model tested to insure a proper installation procedure and to identify potential unforeseen problems. This is the subject of Chapter 8. Chapter 9 covers the area of seakeeping tests. These tests include floating structures moored to the ocean floor by mooring lines, articulated columns, vertically tethered structures, e.g., tension leg platforms and compliant structures. Special care is needed to insure proper duplication of model response without introduction of additional effects through setup. Example cases are discussed and illustrated.

In all these tests, data are recorded by the various instruments installed on the model. These data require special routines to reduce raw recorded data to a usable form for application in a design and analysis. Special care is taken to avoid spurious data entering into the test data. The data analysis techniques that may be used in reducing test results are described in Chapter 10. Examples are taken from specific tests to illustrate these techniques.

It should be noted here that the dimensional units in describing quantities in the book are somewhat mixed. This reflects the slow transition in this country from the English to the metric system. Wherever possible dual units have been provided. In general metric system has been followed. However, with conversion factor noted, some examples have been left in the English units. It is hoped that readers will have appreciation for both systems in following the material in the book.

A list of symbols has been included at the end of the book. Attempt has been made to maintain consistency throughout the book. A few variables have been used with dual meanings in two different parts of the book with little confusion. Some local variables have not been included in the list to limit its size. They have been defined locally where they appear. Subscripts and superscripts have been defined in the list. A list of all the abbreciations appearing in the book has also been included for convenience. 
As is evident from its contents, this book should be a valuable addition to the library of all offshore engineers and naval architects whether they are involved in the research, design, construction or offshore operations. All hydraulic and ocean engineering curriculums in universities offer a course in modeling. This book should be a very appropriate reference for such a course. It is written such that it may be used as a text for a junior or senior level course in a four year engineering curriculum. Since the book deals with many subjects in modeling that go beyond the specifics for offshore structures, it should be found useful by all engineers and scientists interested in structural or hydraulic testing. Finally, it should also be valuable as a reference to many model testing facilities as a complement to their expertise in the area. 
TABLE OF CONTENTS

DEDICATION

ACKNOWLEDGEMENTS ....................................................................... vii

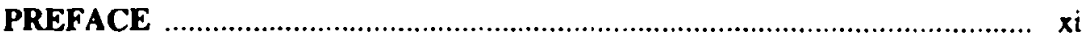

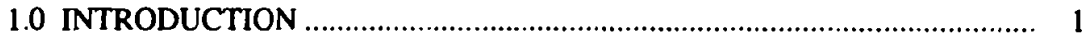

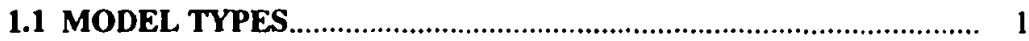

1.2 BRIEF HISTORY OF MODEL TESTING ………............................ 2

1.3 PURPOSE OF MODEL TESTING ................................................ 4

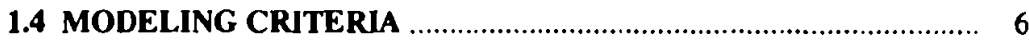

1.5 PLANNING A MODEL TEST.................................................. 9

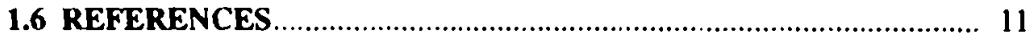

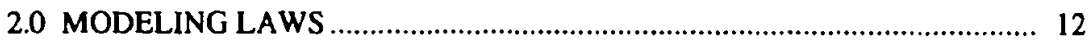

2.1 GENERAL DISCUSSIONS OF SCALING LAWS AND METHODS 12

2.2 BUCKINGHAM PI THEOREM............................................... 14

2.2.1 Dimensionality of Wave Motion ............................................... 17

2.3 NONDIMENSIONAL HYDRODYNAMIC FORCES .......................... 17

2.4 FROUDE'S MODEL LAW ....................................................... 19

2.5 SCALING OF A FROUDE MODEL ............................................. 21

2.5.1 Wave Mechanies Scaling...................................................... 21

2.5.2 Current Drag Scaling ............................................................ 28

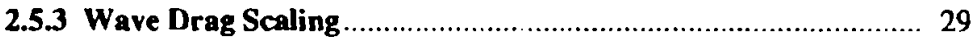

2.6 HYDROELASTIC STRUCTURAL SCALING …….......................... 33

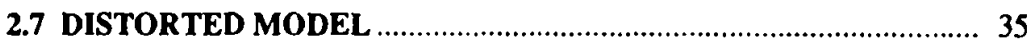

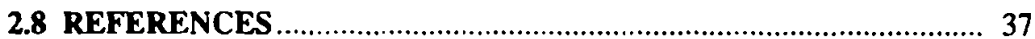

3.0 MODEL CONSTRUCTION TECHNIQUES ……...................................... 40

3.1 GENERAL REQUIREMENTS FOR MODELS ……........................ 40

3.2 MODEL TYPES

3.3 ENVIRONMENTAL LOAD MODELS............................................ 41

3.3.1 OTEC Platform Model ........................................................ 42

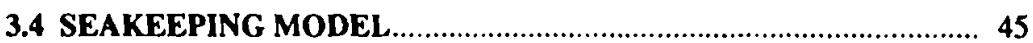

3.4.1 Tanker Model ......................................................................... 47

3.4.1.1 Wood Construction of Model ........................................... 47 
3.4.1.2 Fiberglass Construction of Model......................................... 49

3.4.2 Submergence Model ...................................................................... 50

3.4.2.1 Construction Technique ............................................... 51

3.4.2.2 Static and Dynamic Properties......................................... 53

3.4.3 Tension Leg Platform Model ................................................... 53

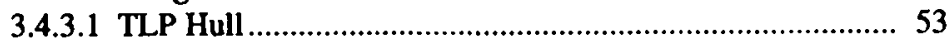

3.4.3.2 Tendons and Tendon Attachment Joints ........................... 55

3.4.3.3 TLP Model Deployment.................................................. 56

3.4.4 Jacket Launching Models ........................................................... 56

3.4.4.1 Jacket Model ................................................................ 56

3.4.4.2 Barge Model .................................................................. 57

3.5 CONSTRUCTION OF A MOORING SYSTEM …......................... 57

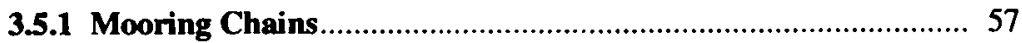

3.5.2 Mooring Hawsers ................................................................. 59

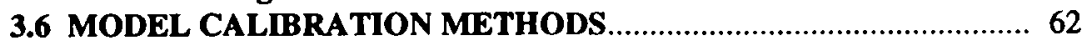

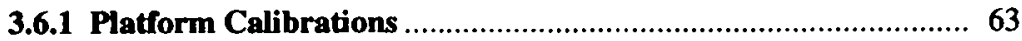

3.6.1.1 Weight Estimate ................................................................. 63

3.6.1.2 Center of Gravity Estimate ................................................ 64

3.6.1.3 Estimate of Moments of Inertia ........................................... 66

3.6.1.4 Righting Moment Calibration ............................................. 66

3.6.2 Tendon Calibrations ......................................................... 67

3.6.2.1 Dry Creep Characteristics................................................... 69

3.6.2.2 Dry Static Stiffness ........................................................... 70

3.6.2.3 Dry Dynamic Stiffness and Damping ................................ 71

3.6.2.4 Hysteresis Effect Under Dry Dynamic Loading................... 71

3.6.2.5 Wet In-Place Static Stiffness ........................................... 71

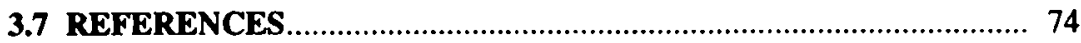

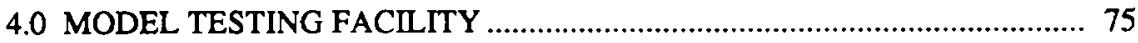

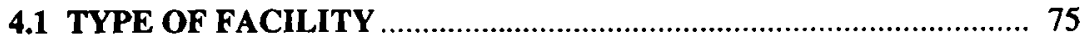

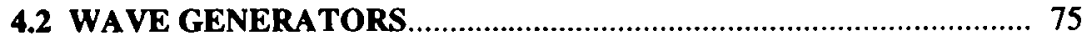

4.3 MECHANICAL WAVEMAKER............................................... 77

4.3.1 Hinged Flapper Wave Theory …………................................. 78

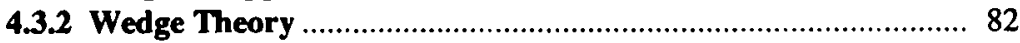

4.4 PNEUMATIC WAVE GENERATOR ............................................ 86

4.5 DESIGN OF A DOUBLE FLAPPER WAVEMAKER ...................... 88

4.5.1 Wetback and Dryback Design ................................................. 90

4.5.2 Hydraulic and Pneumatic Units ............................................. 90

4.5.3 Control System for Two-Board Flapper .................................. 90

4.5.4 Waveboard Sealing and Structural Support System.................. 91

4.6 A TYPICAL WAVE TANK ……................................................. 91 
4.6.1 Low Frequency Wavemaker................................................... 94

4.6.2 High Frequency Wavemaker......................................................... 96

4.7 DESIGN OF MULTIDIRECTIONAL WAVE GENERATOR............ 97

4.7.1 Actuator and Control ..........................................................100

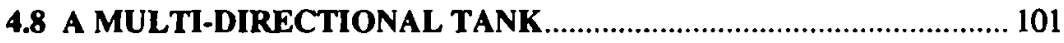

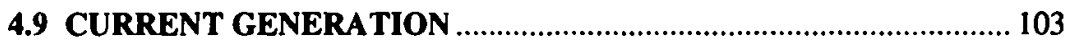

4.9.1 A Typical Current Generator .................................................. 103

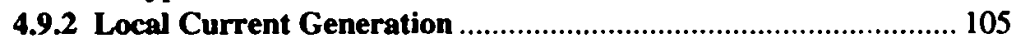

4.9.3 Shear Current Generation ..................................................... 106

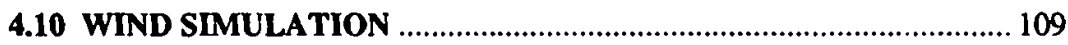

4.11 INSTRUMENTED TOWING STAFF ….......................................... 110

4.12 PLANAR MOTION MECHANISM ............................................. 111

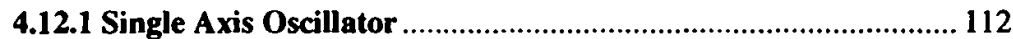

4.13 LABORATORY WA VE ABSORBING BEACHES .............................. 113

4.13.1 Background on Artificial Beaches........................................ 115

4.13.2 Progressive Wave Absorbers ................................................ 117

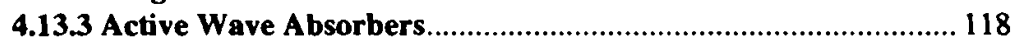

4.13.4 Corrected Wave Incidence......................................................... 118

4.14 REFLECTION OF REGULAR WA VES ...................................... 120

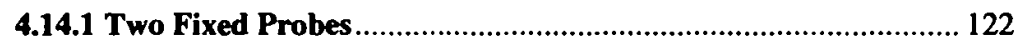

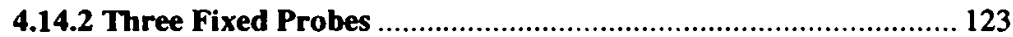

4.15 REFLECTION OF IRREGULAR WAVES ................................... 126

4.16 LIMITED TANK WIDTH............................................................... 128

4.17 TESTING FACILITIES IN THE WORLD ….................................. 130

4.17.1 Institute of Marine Dynamies Towing Tank, St. John's,

Newfoundland, Canada ............................................................. 130

4.17.2 Oftshore Model Basin, Escondindo, California ......................... 131

4.17.3 Offshore Technology Research Center, Texas A\&M University, College Station, Texas ......................................................... 132

4.17.4 David Taylor Research Center, Bethesda, Maryland................ $132^{\circ}$

4.17.5 Maritime Research Institute, The Netherlands (MARIN) ......... 133

4.17.6 Danish Maritime Institute, Lyngby, Denmark ........................... 134

4.17.7 Danish Hydraulic Institute, Horsholm, Denmark ....................... 135

4.17.8 Norwegian Hydrodynamic Laboratory, Trondheim, Norway

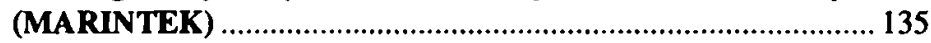

4.18 REFERENCES.

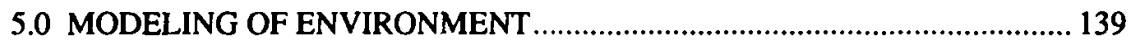

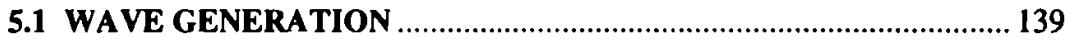

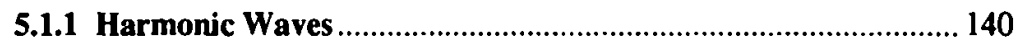


5.1.2 Non-Harmonic Waves.................................................. 140

5.1.3 Imperfect Waves ................................................................. 142

5.1.4 Shallow Water Waves .......................................................... 144

5.2 RANDOM WAVE SIMULATION ............................................... 146

5.2.1 Random Phase Method ....................................................... 147

5.2.2 Random Complex Spectrum Method .................................... 149

5.2.3 Random Coefficient Method................................................. 150

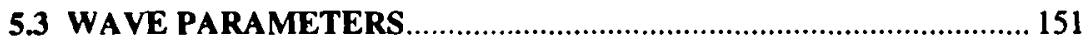

5.3.1 Wave Groups............................................................. 151

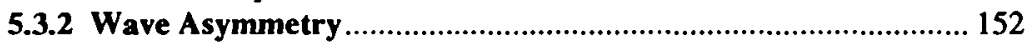

5.3.3 Group Statistics ....................................................... 152

5.4 HIGHER HARMONIC WAVES ............................................ 155

5.5 GENERATION OF MULTI-DIRECTIONAL WAVES.................... 159

5.5.1 Procedure for Simulation of Sea State ..................................... 161

5.5.2 Multi-Directional Generation Theory ................................ 162

5.6 GENERATION OF STEEP WAVES ........................................ 166

5.7 GAUSSIAN WAVE PACKET FOR TRANSIENT WAVES .............. 169

5.8 CURRENT GENERATION .................................................... 173

5.8.1 Wave Current Interaction ............................................. 176

5.8.1.1 Interaction Theory ................................................... 176

5.8.1.2 Combined Wave-Current Tests ................................. 178

5.9 WIND GENERATION ....................................................... 181

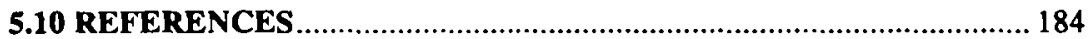

6.0 INSTRUMENTATION AND SIGNAL CONTROL .............................. 190

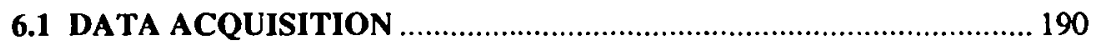

6.1.1 Transducer ..................................................................... 190

6.1.2 Signal Conditioning......................................................... 191

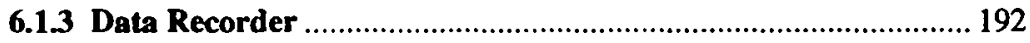

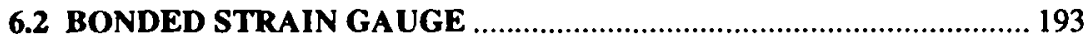

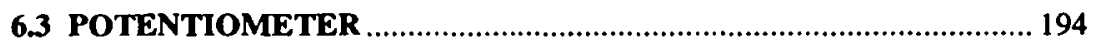

6.4 DISPLACEMENT AND ROTATIONAL TRANSDUCERS .............. 194

6.5 VELOCITY TRA NSDUCERS ............................................. 196

6.6 ONE DIMENSIONAL CURRENT PROBE................................... 197

6.7 TWO DIMENSIONAL CURRENT PROBE.................................. 198

6.7.1 Basic Properties................................................................. 198

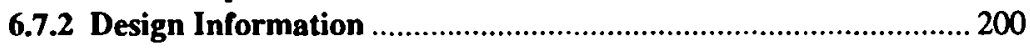

6.7.3 Calibration and Testing ........................................................ 201

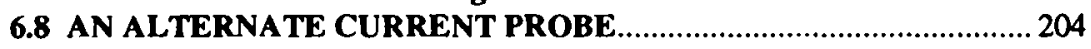

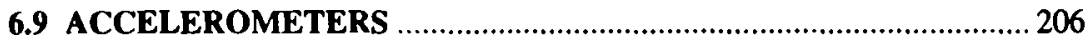




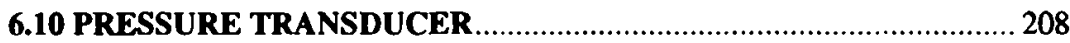

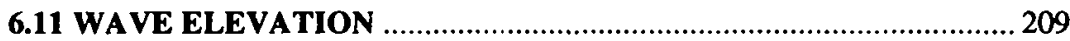

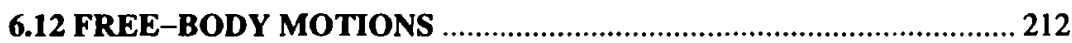

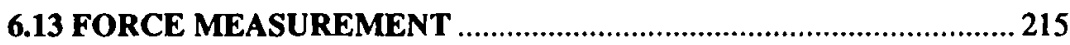

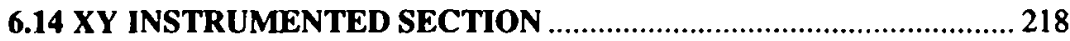

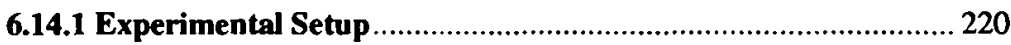

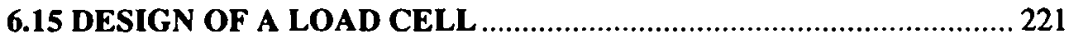

6.15.1 Shear Force and Bending Moment.......................................... 222

6.15.2 Layout of Strain Gauges ........................................................... 223

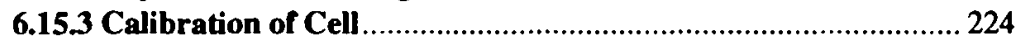

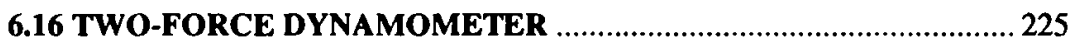

6.17 TOWING STAFF INSTRUMENTATION .......................................... 226

6.18 MECHANICAL OSCILLATION OF A FLOATING BODY ............. 227

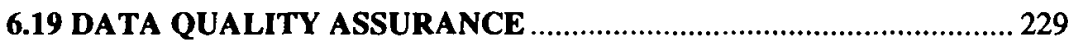

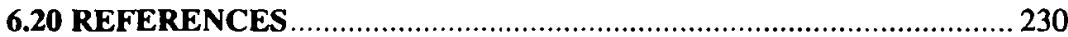

7.0 MODELING OF FIXED OFFSHORE STRUCTURES …...............................2 232

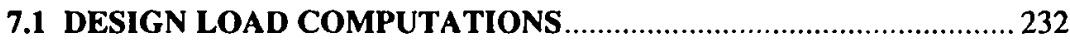

7.2 SMALL-MEMBERED FIXED STRUCTURES ….............................233

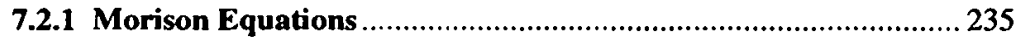

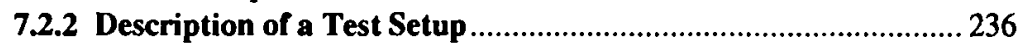

7.2.3 Pressure Profile Around a Cylinder ........................................... 240

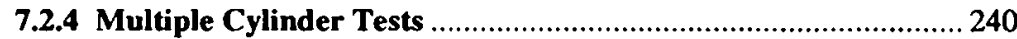

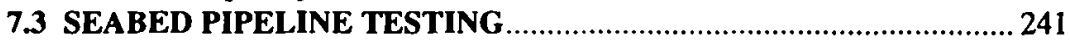

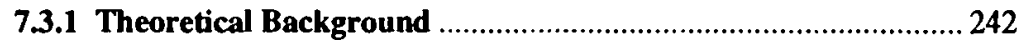

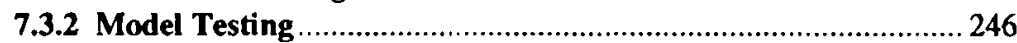

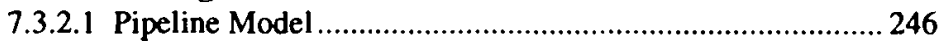

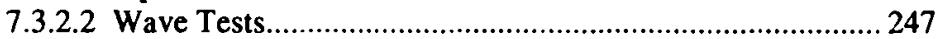

7.3.2.3 Force Servo-Control Mechanism ........................................ 249

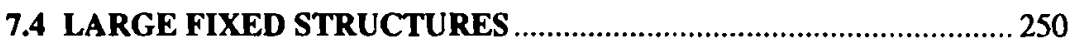

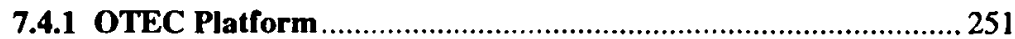

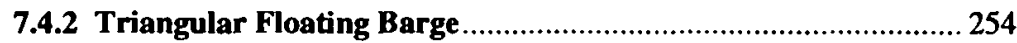

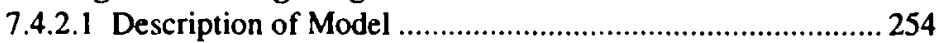

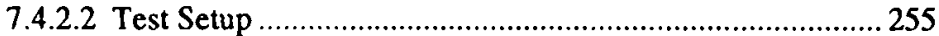

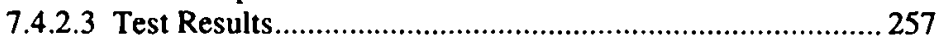

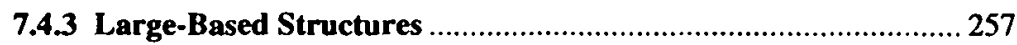

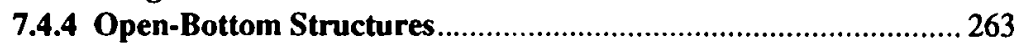

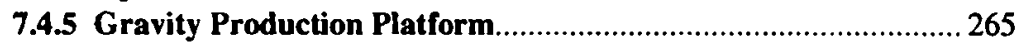

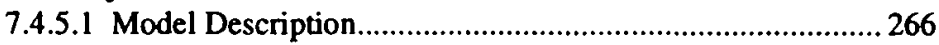

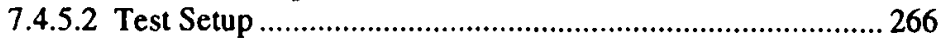




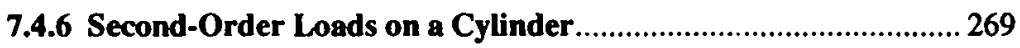

7.4.7 Scaling of Fixed Elastic Structures.............................................. 277

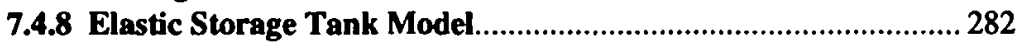

7.4.8.1 Elastic Model Design ........................................................ 283

7.4.8.2 Elastic Model Tests............................................................ 286

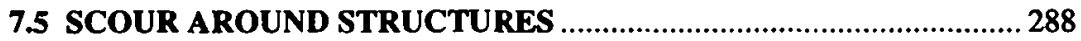

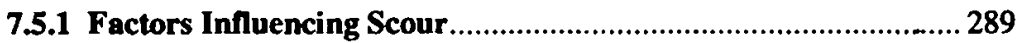

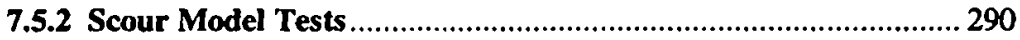

7.5.3 Scaling of Soil-Structure Interaction ..........................................292

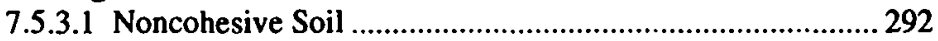

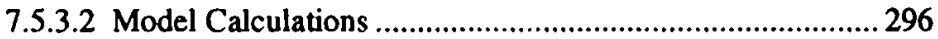

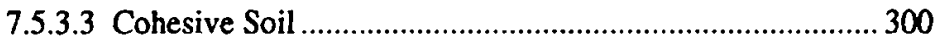

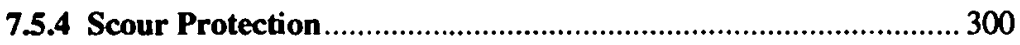

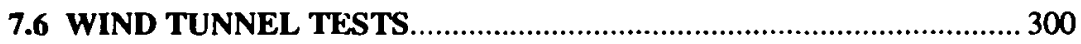

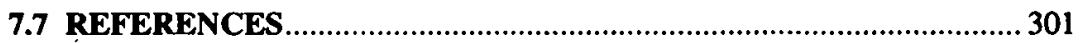

8.0 MODELING OF OFFSHORE OPERATIONS …….........................................

8.1 TYPES OF OFFSHORE OPERATIONS ……...................................... 305

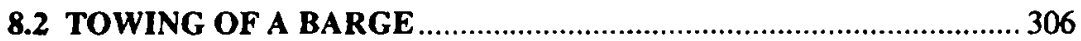

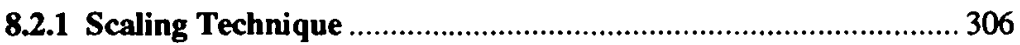

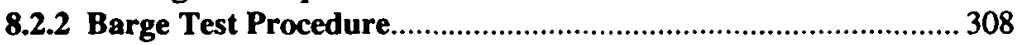

8.2.3 Data Analysis and Results..........................................................309

8.3 SUBMERSIBLE DRILLING RIG TOWING TESTS …..................... 312

8.3.1 Test Description ............................................................................ 313

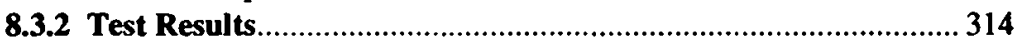

8.4 TOWING OF A BUOYANT TOWER MODEL …................................ 315

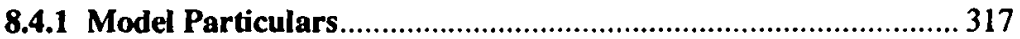

8.4.2 Towing Tests.............................................................................. 317

8.4.3 Bending Moment Tests ………............................................. 319

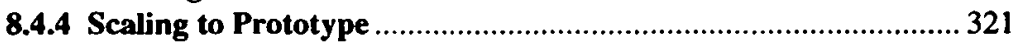

8.5 LAUNCHING OF OFFSHORE STRUCTURES ….......................... 323

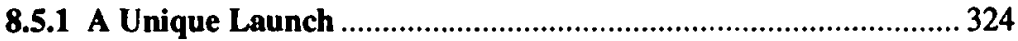

8.5.1.1 Modeling of Soft-Volume Cans........................................ 324

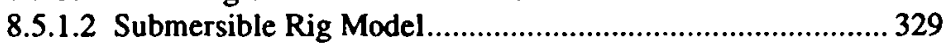

8.5.1.3 Launching of Mat on Cans ................................................ 332

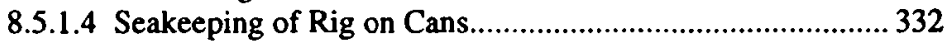

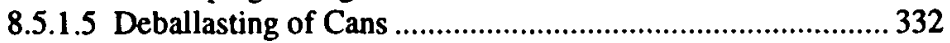

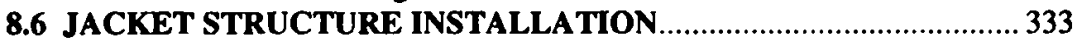

8.6.1 Scaling of Jacket Installation Parameters................................... 333

8.6.2 Launching Test Procedure........................................................... 335 
8.6.3 Side Launching of Structures ......................................................339

8.7 STAGED SUBMERGENCE OF A DRILLING RIG........................... 339

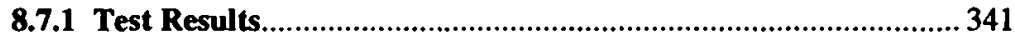

8.8 DYNAMIC SUBMERGENCE OF A SUBSEA STORAGE TANK …. 342

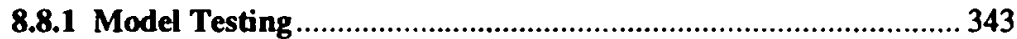

8.9 OFFSHORE PIPE LA YING OPERATIONS ………...........................345

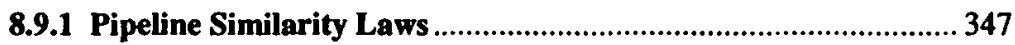

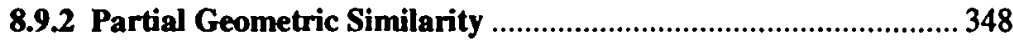

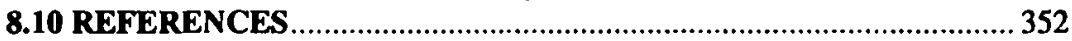

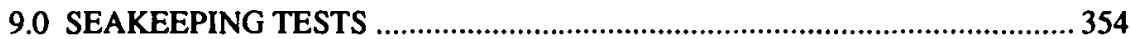

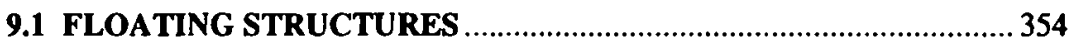

9.2 METHOD OF TESTING FLOATING STRUCTURES ….................. 355

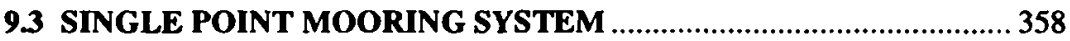

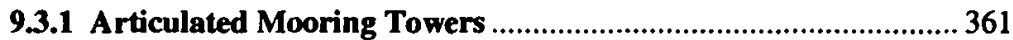

9.4 TOWER-TANKER IN IRREGULAR WA VES .......................................369

9.5 TESTING OF A FLOATING VESSEL .............................................370

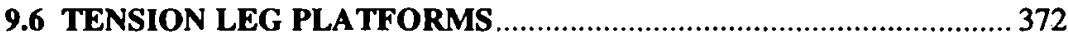

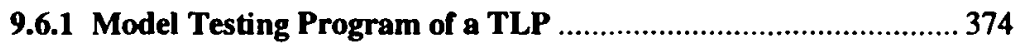

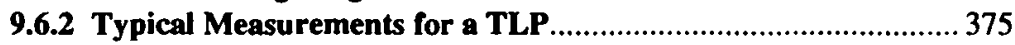

9.6.3 Wave Frequency Response of a TLP........................................375

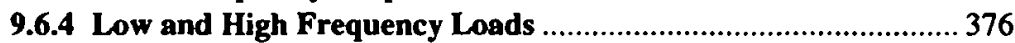

9.7 DRIFT FORCE TESTING OF A MOORED FLOATING VESSEL ... 381

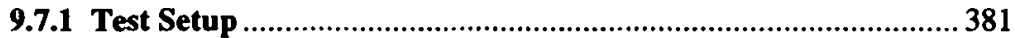

9.7.2 Hydrodynamic Coefficients at Low Frequencies......................... 382

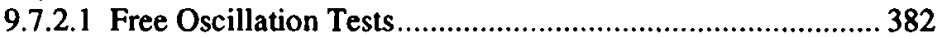

9.7.2.2 Forced Oscillation Tests.................................................. 382

9.8 DAMPING COEFFICIENTS OF A MOORED FLOATING VESSEL382

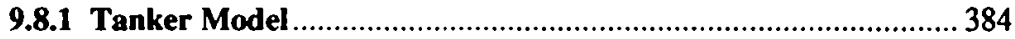

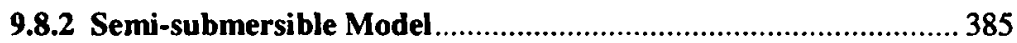

9.8.3 Heave Damping of a TLP Model ................................................387

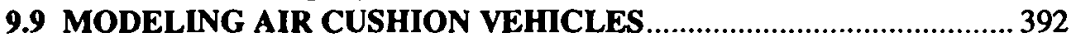

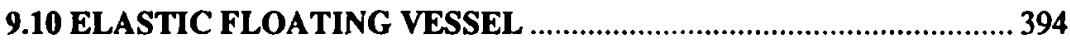

9.11 MODELING OF A LOADING HOSE ……….................................395

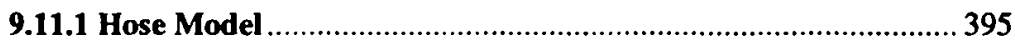

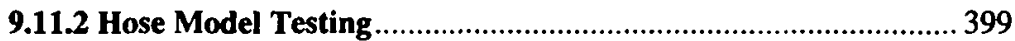

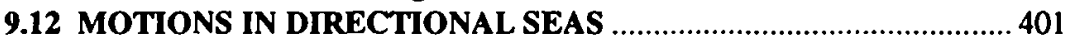

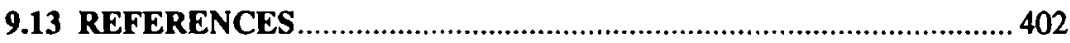


10.0 DATA ANALYSIS TECHNIQUES....................................................... 406

10.1 STANDARD DATA ANALYSIS ........................................... 406

10.2 REGULAR WAVE ANALYSIS ................................................ 408

10.2.1 Standing Wave .................................................................. 409

10.2.2 Reflected Wave...................................................................... 410

10.2.3 Spurious Wave Data........................................................... 410

10.3 IRREGULAR WAVE ANALYSIS........................................... 411

10.3.1 Fourier Series Analysis ....................................................4 411

10.3.2 Wave Spectrum Analysis .................................................... 412

10.3.3 Wave Group Analysis ..................................................... 414

10.3.4 Statistical Analysis .......................................................... 416

10.4 ANALYSIS OF DIRECTIONAL WAVES ................................ 417

10.5 FILTERING OF DATA .............................................................. 419

10.6 RESPONSE ANAL YSIS ..................................................... 422

10.6.1 Frequency Domain Analysis ............................................. 423

10.6.2 Linear System ................................................................. 427

10.6.3 Theory of Cross Spectral Analysis ...................................... 429

10.6.4 Error Analysis .............................................................. 432

10.6.5 Example Problem ..............................................................434

10.6.6 Nonlinear System ................................................................. 438

10.7 ANALYSIS OF WA VE FORCE COEFFICIENTS ........................4 439

10.7.1 Fourier Averaging Method ........................................... 441

10.7.2 Least Square Technique ................................................ 443

10.8 FREE VIBRATION TESTS ..................................................... 445

10.8.1 Low Frequency Hydrodynamic Coefricients .......................... 445

10.8.1.1 Linear System ............................................................. 445

10.8.1.2 Nonlinear System ........................................................ 449

10.8.2 Mechanical Oscillation......................................................... 451

10.8.3 Random Decrement Technique ........................................... 456

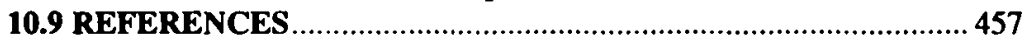

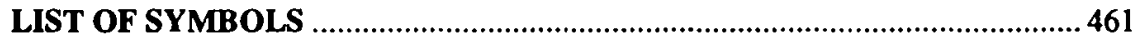

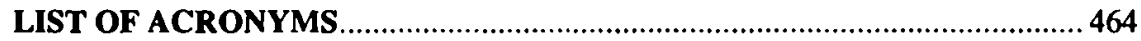

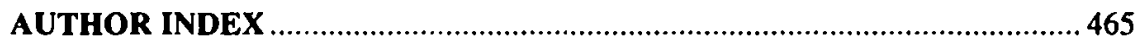

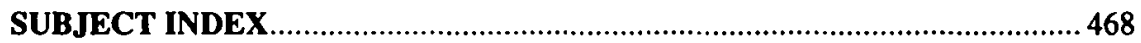




\section{OFFSHORE STRUCTURE MODELING}

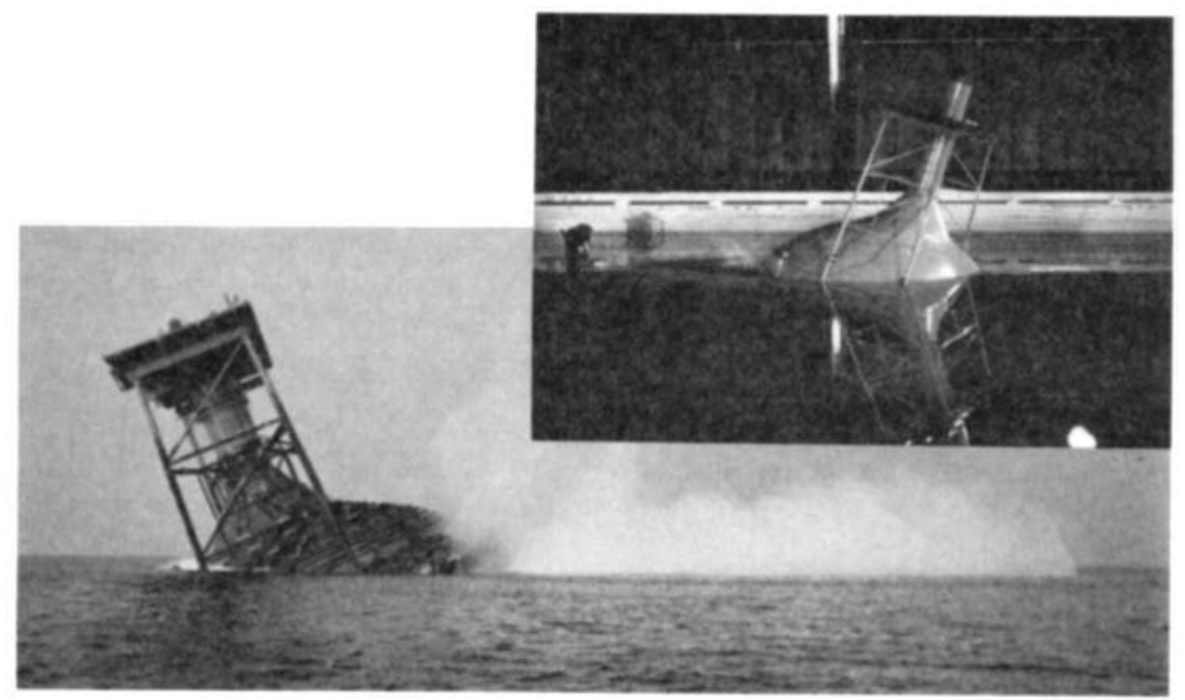

"I contend that unless the reliability of small scale experiments is emphatically disproved, it is useless to spend vast sums of money on full scale trials which afterall may be misdirected unless the ground is thoroughly cleared beforehand by an exhaustive investigation on a small scale."

William Froude, 1886 